\title{
Size-Dependent Photocatalytic Activity of Cubic Boron Phosphide Nanocrystals in the Quantum Confinement Regime
}

Hiroshi Sugimoto, ${ }^{* \dagger}$ Bálint Somogyi, ${ }^{*}$ Toshiyuki Nakamura, ${ }^{\dagger}$, Hao Zhou, ${ }^{\dagger}$ Yuichi Ichihashi, ${ }^{\S}$ Satoru Nishiyama, ${ }^{\S}$ Adam Gali ${ }^{\ddagger} \perp$ and Minoru Fujii ${ }^{\dagger}$

${ }^{\dagger}$ Department of Electrical and Electronic Engineering, Graduate School of Engineering, Kobe University, Rokkodai, Nada, Kobe 657-8501, Japan

${ }^{\ddagger}$ Institute for Solid State Physics and Optics, Wigner Research Centre for Physics, Hungarian Academy of Sciences, P.O. Box 49, H-1525 Budapest, Hungary ${ }^{\S}$

Department of Chemical Science and Engineering, Graduate School of Engineering, Kobe University, Rokkodai, Nada, Kobe 657-8501, Japan

${ }^{\perp}$ Department of Atomic Physics, Budapest University of Technology and Economics, Budafoki út 8, H-1111 Budapest, Hungary 


\section{Performance of PBE and PBE0 functionals for BP}

As cubic and especially hexagonal BP are not as widely studied materials as silicon or carbon, we performed some benchmark calculations in order to gain some insight about the accuracy of the $\mathrm{PBE}^{1}$ and $\mathrm{PBE}^{2}$ functionals. It is important to keep in mind that conclusion of the benchmarks carried out for bulk materials cannot be straightforwardly applied to nanostructures. For example, PBE0 was found the underestimate the HOMO-LUMO gap of very small Si nanoparticles ${ }^{3}$, while it is known to overestimate the band gap in the bulk limit ${ }^{4}$. These benchmarks are still expected to be useful as the size of the produced BP nanostructures is rather large, where quantum confinement becomes weak. Our benchmarks included the PBE (GGA) functional, the $\mathrm{SCAN}^{5}$ (METAGGA), the PBE0 hybrid functional and the HSE0 $6^{6}$ screened-hybrid functional. For bulk cubic BP, we obtained $1.24 \mathrm{eV}, 1.18 \mathrm{eV}$, $2.66 \mathrm{eV}$ and $1.98 \mathrm{eV}$ indirect gaps for the PBE, SCAN, PBE0 and HSE06 functionals, respectively. The experimental band gap of BP is 2.0-2.1 eV. The cost-effective PBE and SCAN severely underestimate the gap of BP, while the range-separated hybrid HSE06 provides the best results. On the other hand, the PBE0 hybrid-functional significantly overestimates the gap of cubic BP in the bulk limit, which might be relevant for our results of larger BP nanocrystals.

For a single hexagonal BP layer, we obtained $0.87 \mathrm{eV}, 0.94 \mathrm{eV}, 1.68 \mathrm{eV}$ and $1.29 \mathrm{eV}$ direct band gaps for the PBE, SCAN, PBE0 and HSE06 functionals, respectively. The experimental band gap of hexagonal BP is not known, but high accuracy GW calculations ${ }^{7}$ indicate $1.81 \mathrm{eV}$ gap for a single monolayer.

\section{Calculation of the radiative lifetimes, application of scissor operator}

Adiabatic linear-response TDDFT calculations become very expensive with increasing nanocrystal size. In addition, the energy spacing between the first few excitations becomes comparable with the thermal energy, thus simply calculating only the first vertical excitation energy is not enough. Because of this, the transition dipole moments of low energy vertical excitations were obtained within the independent particle approximation for Kohn-Sham states obtained by VASP (PBE) calculations. The radiative transition rate between the KohnSham orbitals indexed by $i$ and $j$ are obtained according to the Weisskopf-Wigner theory:

$$
\Gamma_{i j}=\frac{n E_{i j}^{3} \mu_{i j}^{2}}{3 \pi \epsilon_{0} \hbar^{4} c^{3}}
$$

where $n$ is the refractive index ( $n=1$ for nanoparticles in vacuum), $E_{i j}$ is the transition energy, $\mu_{i j}$ is the transition dipole moment, $\varepsilon_{0}$ is the vacuum permittivity and $c$ is the speed of light. The thermally averaged total rate is calculated by 


$$
<\Gamma>_{T}=\frac{\sum_{i, j} \Gamma_{i j} e^{-E_{i j} / k_{b} T}}{\sum_{i, j} e^{-E_{i j} / k_{b} T}} .
$$

Finally, the radiative lifetime is obtained as the inverse of the thermally averaged transition rate. We note that the independent particle approximation utilizing the PBE functional introduces some errors: the PBE underestimates the gap, while the independent particle picture neglects the Coulomb interaction between the electron-hole pair. To alleviate these issues, we introduce simple correction based on adiabatic linear-response TDDFT calculations for the smaller nanocrystals. Table S1 shows the calculated PBE/PBE0 band gaps and vertical excitation energies for all model nanocrystals.

\begin{tabular}{|c|c|c|c|c|c|}
\hline Diameter (nm) & Gap (PBE) & Gap (PBE0) & $\begin{array}{c}\text { Vertical Excitation } \\
\text { (PBE0) }\end{array}$ & $\begin{array}{c}\text { Exciton energy } \\
\text { (PBE0) }\end{array}$ & $\begin{array}{c}\text { Gap difference } \\
\text { PBE0-PBE }\end{array}$ \\
\hline 1.28 & 2.33 & 3.94 & 3.39 & 0.55 & 1.61 \\
\hline 1.62 & 2.02 & 3.65 & 3.13 & 0.53 & 1.63 \\
\hline 1.71 & 1.74 & 3.24 & 2.88 & 0.36 & 1.49 \\
\hline 1.83 & 1.84 & 3.48 & 3.03 & 0.45 & 1.65 \\
\hline 1.89 & 1.52 & 2.97 & 2.61 & 0.36 & 1.45 \\
\hline 2.07 & 1.46 & 2.81 & - & - & 1.35 \\
\hline 2.36 & 1.71 & 3.14 & - & - & 1.43 \\
\hline 2.84 & 1.19 & - & - & - & - \\
\hline
\end{tabular}

Table S1. Calculated PBE/PBE0 gaps and the lowest vertical-excitation energies. We define the exciton energy as the difference between the gap and the vertical lowest vertical excitation energy. Some values were not calculated due to large computational costs. All energy values are given in $\mathrm{eV}$.

Our correction scheme is the following: we assume that the exciton binding energy is the same for all electron-hole pairs of a given nanocrystal, and PBE underestimates the energy of all unoccupied states of a given model nanocrystal by the same amount due to the band gap error. (neither of these assumptions are true in principle, but are expected to serve as a suitable approximation that results in radiative lifetimes with correct orders of magnitude). Next, the energies of the unoccupied Kohn-Sham orbitals are increased by the PBE/PBE0 gap difference $(\sim 1.4-1.6 \mathrm{eV})$ and reduced by the exciton binding energy $(\sim 0.4 \mathrm{eV})$. For the largest nanocrystals, where PBE0 calculations are not available, the values for the correction were taken from the largest nanocrystal where both values are available. 
The value of the scissor operator for the corrected energy level structure (Figure 2. in the Manuscript) were calculated similarly, where the value of $1.4 \mathrm{eV}$ is based on the three largest BP nanocrystal model where both the PBE and PBE0 gaps are available.

\section{Electronic structure of BP nanoparticles and the significance of polarized surfaces}

We find that the formation energy and electronic structure of the generated BP nanoparticles does not show a simple size dependence. The calculated trend seemingly goes against the quantum confinement effect (QCE), where one expects monotonously increasing HOMO-LUMO gap with decreasing nanoparticle size. Figure S1a shows the HOMO and LUMO levels versus the BP nanocrystal diameter for our model nanoparticles, calculated in vacuum and in an effective water medium. It is apparent that the some of the nanocrystals are almost metallic in vacuum. In addition, we found that the formation energy of these nanocrystals is higher compared to the ones with larger band gaps. To quantify this, we fit the formation energy of large-gap nanocrystals by $E=c_{1} \cdot n(\mathrm{BP})+c_{2} \cdot n(\mathrm{H})$ where $n(\mathrm{BP})$ and $n(\mathrm{H})$ represents the number of $\mathrm{BP}$ pairs and $\mathrm{H}$ atoms of the nanocrystal. We compared the total energy of the BP nanocrystals with the total energy predicted by this simple model, and plotted the difference in Figure S2b. The two nanocrystal models with drastically reduced band gaps have almost $15 \mathrm{eV}$ larger formation energy than expected based on their size. We found, that this is due to the presence of polarized, B- or P-terminated (111) facets of the BP nanocrystals. 


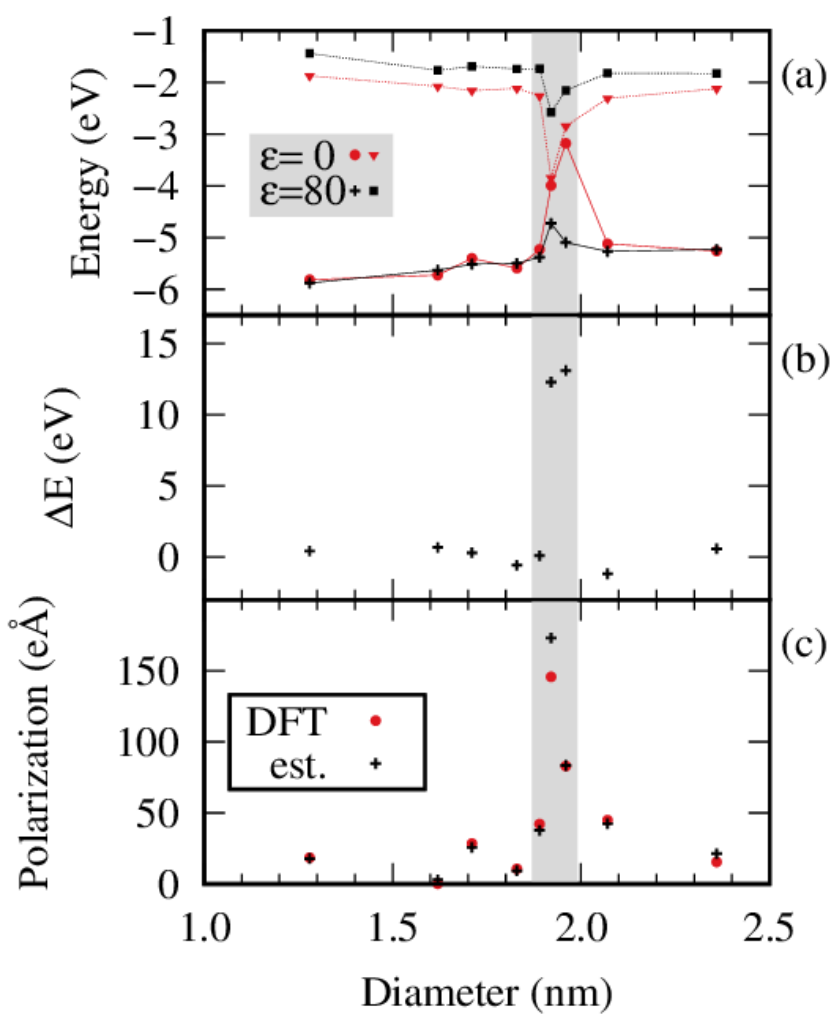

Figure S1. Electronic structure of BP nanocrystals. The shaded gray area highlights three nanoparticles with very similar sizes, but very different electronic structures. (a) The PBE0 HOMO and LUMO energies calculated in vacuum and in water. (b) Difference between the predicted and calculated formation energies. (c) Calculated total dipole moments and estimated dipole moments of the BP nanoparticles.

In particular, the total dipole moment of the BP nanoparticle proved to be a good indicator for the stability: according to our results, BP NPs with small dipole moment are more stable and have larger band gaps compared to BP NPs with larger dipole moments. Figure S1 (c) shows the calculated dipole moments for the BP nanocrystals. Nanoparticles with smaller gaps have large dipole moments. We also calculated the dipole moments by approximating each $\mathrm{B}$ and $\mathrm{P}$ atoms by a $-q$ and $+q$ point charge, respectively. The obtained dipole moments have good agreement with the ones obtained by DFT, indicating a simple correlation between the geometry of the nanocrystals and their dipole moment.

Placed in an aqueous solvent, the effects of surface polarization is somewhat diminished. While the HOMO-LUMO gaps of polarized BP nanocrystals remain smaller compared to BP nanocrystals with smaller polarization, the gap opens from $<0.5 \mathrm{eV}$ to $>2 \mathrm{eV}$. Due to its high dielectric constant, water is able to compensate the polarized surfaces of BP NPs. We also note that aqueous environment also opens up the gap of the other BP nanocrystals too, 
increasing the energy of the LUMO by $\sim 0.5 \mathrm{eV}$.

Because of their large formation energies, we left out the two models with small HOMOLUMO gaps (large dipole moments) from our analysis in the main text. We note however, that the formation process of BP nanoparticles is not very understood, thus the occurrence very polarized BP nanocrystals in experimental samples cannot be ruled out. On the other hand, the shape of realistic nanocrystals is much less symmetric than our models, and the irregularities may prevent the BP nanocrystals from having large dipole moments.

Finally, we investigate the effect of polarized surfaces on the transition rates between the HOMO and LUMO. In Figure 3 of the main text, we demonstrate that the radiative lifetime does not change monotonously with the size of the BP nanocrystals, even after we removed the two previously highlighted nanocrystals model which exhibited very different electronic structure compared to the rest of the models. We choose the $1.62 \mathrm{~nm}$ and $1.71 \mathrm{~nm} \mathrm{BP}$ nanocrystals to illustrate our point, which have very different radiative lifetimes ( $5 \mu$ s and 125 $\mu \mathrm{s}$, respectively) despite their similar sizes. Figure S2 shows the HOMO and LUMO orbitals for both BP nanocrystals. For the smaller BP nanocrystal, the HOMO is delocalized like a bulk-like band, while LUMO is localized on the surface. The HOMO and LUMO are localized on the opposing (B- and P-terminated) facets of the slightly larger nanocrystal, resulting in a minimal overlap between them.
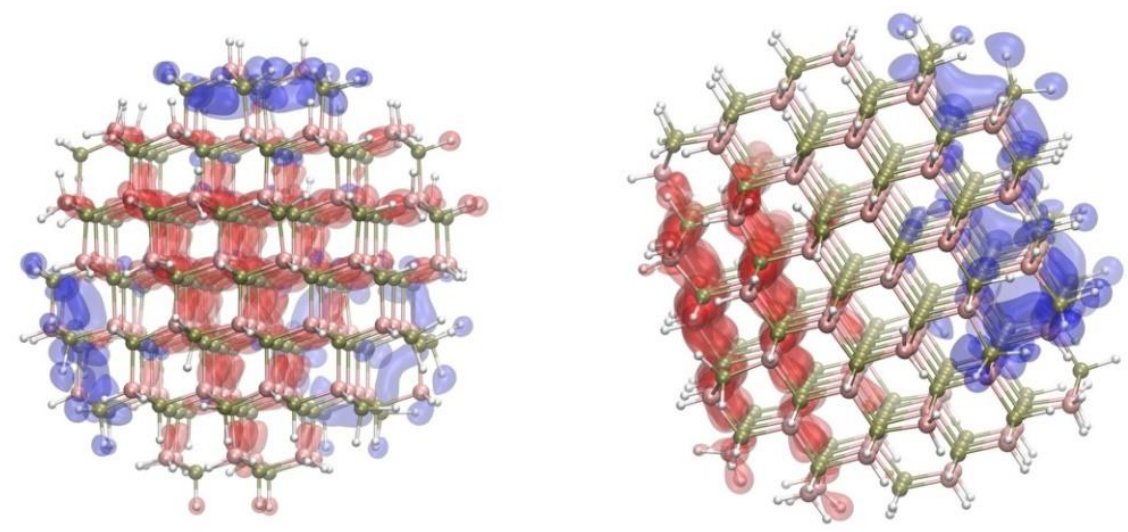

Figure S2. The HOMO (red clouds) and LUMO (blue clouds) of the $1.62 \mathrm{~nm}$ (left) and the $1.71 \mathrm{~nm}$ (right) BP nanocrystal models. Despite the similar size, the shape and localization of the HOMO orbital is qualitatively different for the two nanocrystals resulting in very different radiative lifetimes. 


\section{HOMO and LUMO orbitals in vacuum and water}

In Figure S2, we plotted the HOMO of LUMO orbitals of a (B)-H and (B)-OH terminated $\mathrm{BP}$ nanocrystal calculated in vacuum and in an effective aqueous environment. For the (B)-H terminated nanocrystal, the HOMO and LUMO are localized on B- and P-terminated facets of the nanocrystal. In water, this surface-localization becomes less pronounced, as the HOMO and LUMO orbitals are more constrained towards the core of the nanocrystal due to the high dielectric constant of water. For the (B)-OH terminated BP nanocrystal, the HOMO is localized on a (B)-OH terminated surface while the LUMO on a P-terminated surface. In aqueous environment, the LUMO moves towards the core of the nanocrystal, similarly to the (B)-H terminated case. The HOMO orbitals, on the other hand, remain almost identical in vacuum and in water.

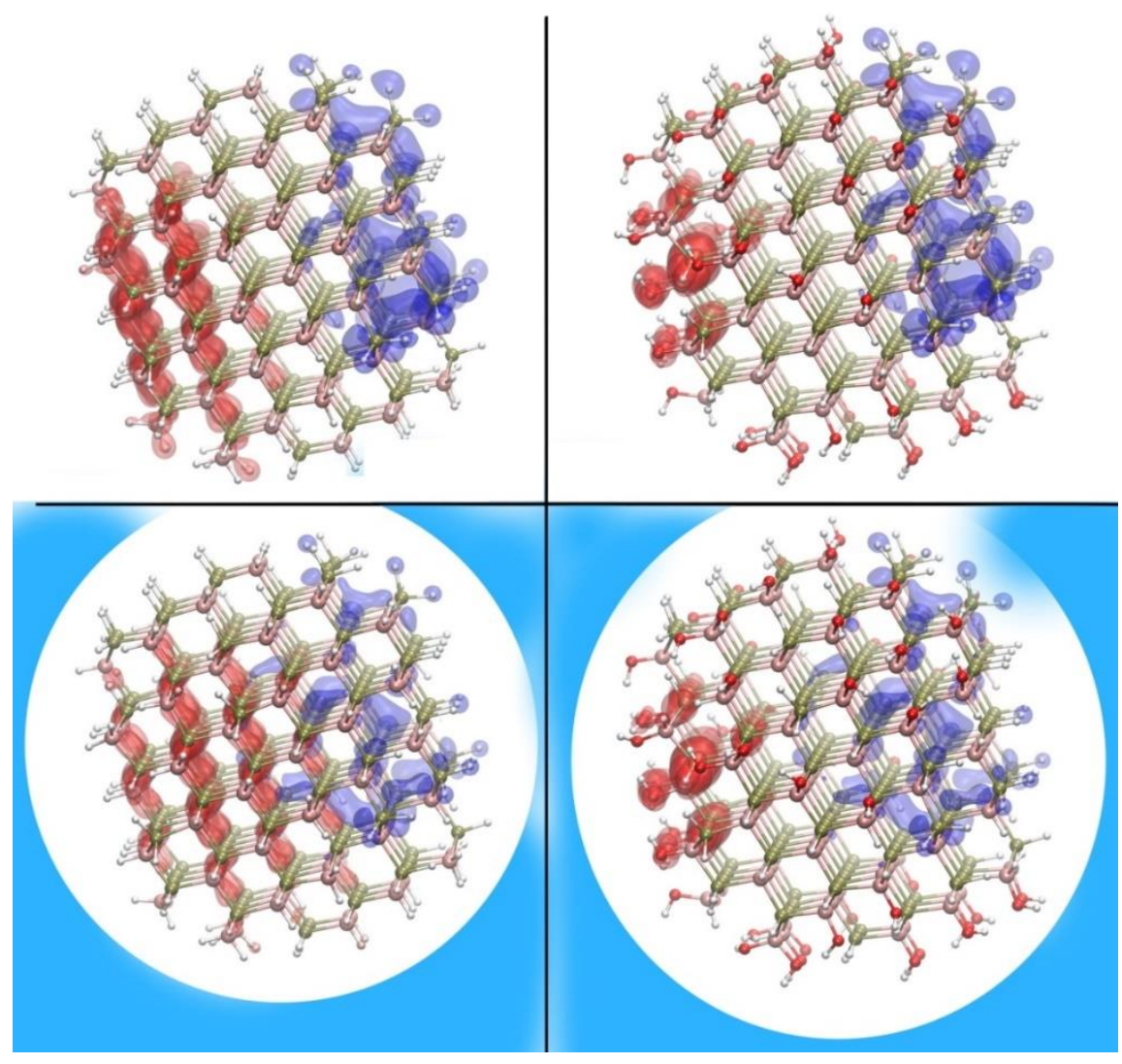

Figure S3. The HOMO (red clouds) and LUMO (blue clouds) orbitals of a $1.71 \mathrm{~nm} \mathrm{BP}$ nanocrystal. The top two pictures represent calculations in vacuum, while the bottom two pictures represent calculations with an effective aqueous model. The left two pictures depict a nanoparticle with (B)-H surface termination, while the left two pictures show a nanoparticle with (B)-OH surface termination. 


\section{Alignment between the $\mathrm{H}_{2} / \mathrm{H}^{+}$level and $\mathrm{CBM}$ of $\mathrm{BP}$}

To calculate the alignment between the CBM of cubic BP and the $\mathrm{H}_{2} / \mathrm{H}^{+}$level, we follow the method of $\mathrm{Wu}$ et al. ${ }^{8}$, which references the band energies to the Hartree potential. The energy difference is obtained as:

$$
E_{\mathrm{CBM}, \text { edge }}-A_{\text {edge }}=\left(E_{\mathrm{CBM}, \text { bulk }}-H_{\text {sc,bulk }}\right)-\left(A_{\text {bulk }}-H_{\text {sol,bulk }}\right)+\left(H_{\text {sc,edge }}-H_{\text {sol,edge }}\right)
$$

where $E_{\mathrm{CBM}}$, bulk/edge are the conduction band minima in the bulk semiconductor and at the semiconductor-water interface, $A_{\text {bulk/edge }}$ are the acceptor level $\left(\mathrm{H}^{+} / \mathrm{H}_{2}\right.$ level $)$ of bulk water and water at the semiconductor interface, and $H_{\mathrm{sc} / \mathrm{sol} \text {,bulk/edge }}$ are the Hartree potentials of bulk semiconductor/water and semiconductor/water at the semiconductor-water interface. All the calculations were performed by the VASP code, utilizing the PBE functional. $A_{\text {bulk }}-H_{\text {sol,bulk }}$ was already determined to be $-0.7 \mathrm{eV}^{8}$, and a simple bulk calculation with a $12 \times 12 \times 12$ Monkhorst-Pack k-point grid resulted in $8.72 \mathrm{eV}$ value for $E_{\mathrm{CBM} \text {,bulk }}-H_{\text {sc,bulk }}$ of cubic boronphosphide. The most challenging step is the determination of $H_{\text {sc,edge }}-H_{\text {sol,edge. We constructed }}$ a slab model consisting $288 \mathrm{~B} / \mathrm{P}$ atoms and 144 water molecules, depicted in Figure S4. Slabs with (100) or (111) surface orientation lead to a B and P terminated surfaces, and the macroscopic polarization results in metallic electronic structure. Because of this, our slab model was created with (110) surface orientation, which ensures that both sides of the slab are terminated with $1: 1$ ratio of $B$ and $P$.

Before the addition of water, the slab structure was relaxed by DFT. After the vacuum between the slabs was filled by water molecules of appropriate density, we performed a canonical (nVT) molecular dynamics (MD) simulation utilizing the LAMMPS code $^{9}$ with the TIP4P ${ }^{10}$ rigid water model. After an initial run of $125 \mathrm{ps,} \mathrm{we} \mathrm{created} 31$ snapshots of the geometry with $12.5 \mathrm{ps}$ intervals between them. The overall MD run lasted for $500 \mathrm{ps}$. We calculated $H_{\text {sc,edge }}-H_{\text {sol,edge }}$ for each snapshot by DFT, using only the $\Gamma$-point. After averaging, we determined $H_{\text {sc,edge }}-H_{\text {sol,edge }}=-9.23 \mathrm{eV} \pm 0.19 \mathrm{eV}$, where the error is the standard deviation of the obtained values. These values provide $-0.2 \mathrm{eV}$ (vs. NHE) for the CBM of cubic BP. We note that the statistical error of our calculation in itself is $\sim 0.2 \mathrm{eV}$, and we do not discuss additional sources of error, such as DFT(PBE)-related errors or the potential error originating from the finite size of the system. Figure S5 depicts the planar averages of the Hartree potential calculated alongside of the axis perpendicular to the slab surface for two of our 31 snapshots. We show the two snapshots with the minimal and maximal values for $H_{\text {sc,edge }}-$ $H_{\text {sol,edge. }}$ 


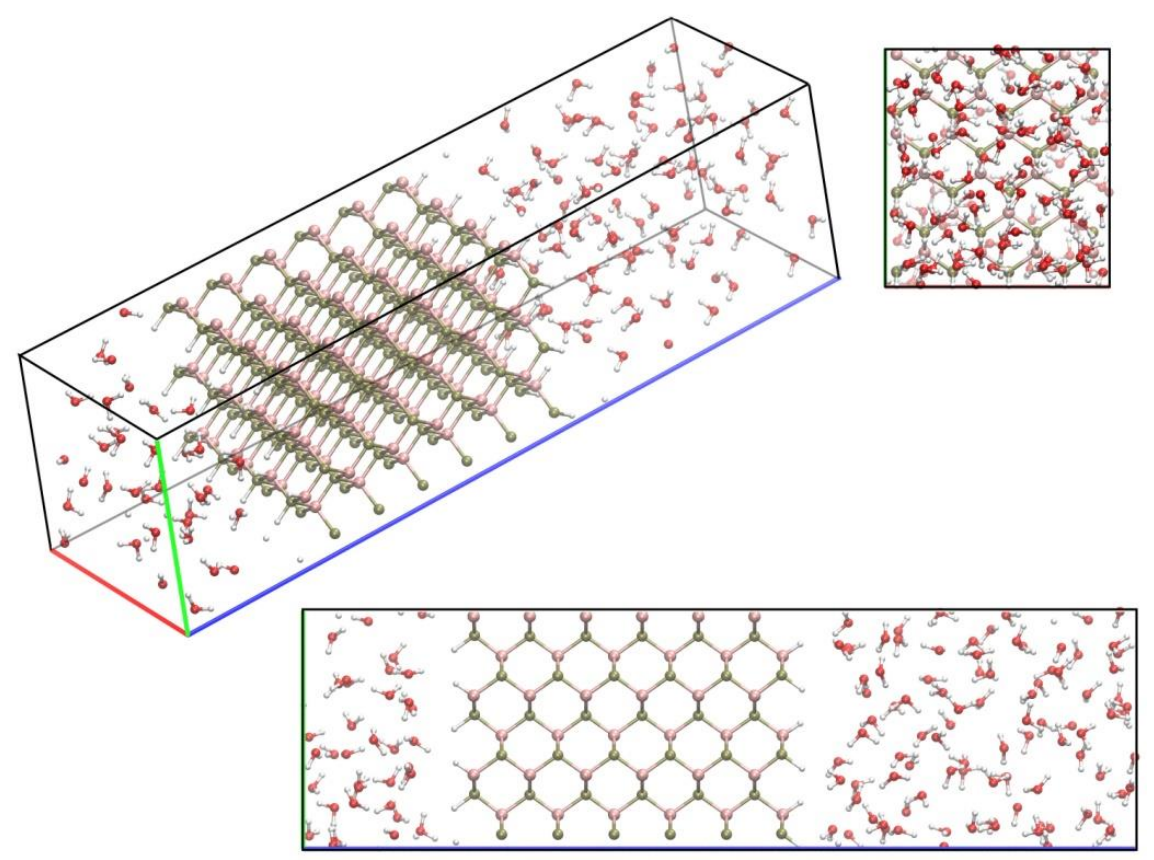

Figure S4. The geometry of our BP slab + water model from 3 different perspectives.

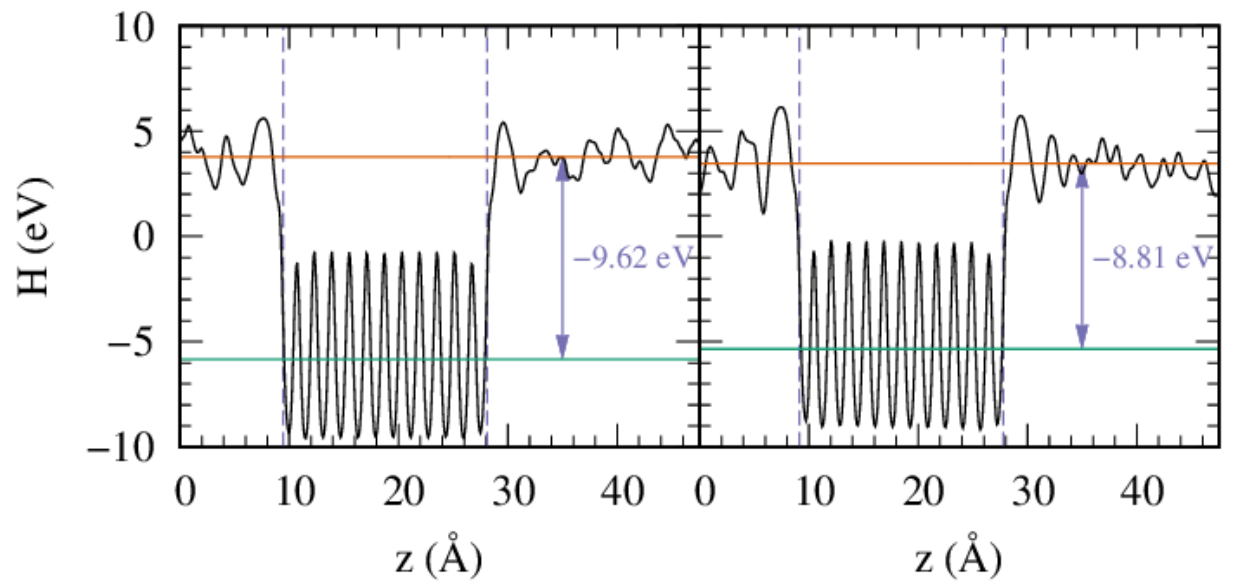

Figure S5. Calculated planar averages of the Hartree potential for the BP slab + water model, in the direction perpendicular to the $\mathrm{BP} /$ water interface. The left and right graphs show the results for the snapshots with the largest and smallest absolute values for $H_{\text {sc,edge }}-H_{\text {sol,edge }}$. Orange and green horizontal lines show the average potential of water and the BP slab, respectively. Vertical dashed lines mark the semiconductor-water interface. 

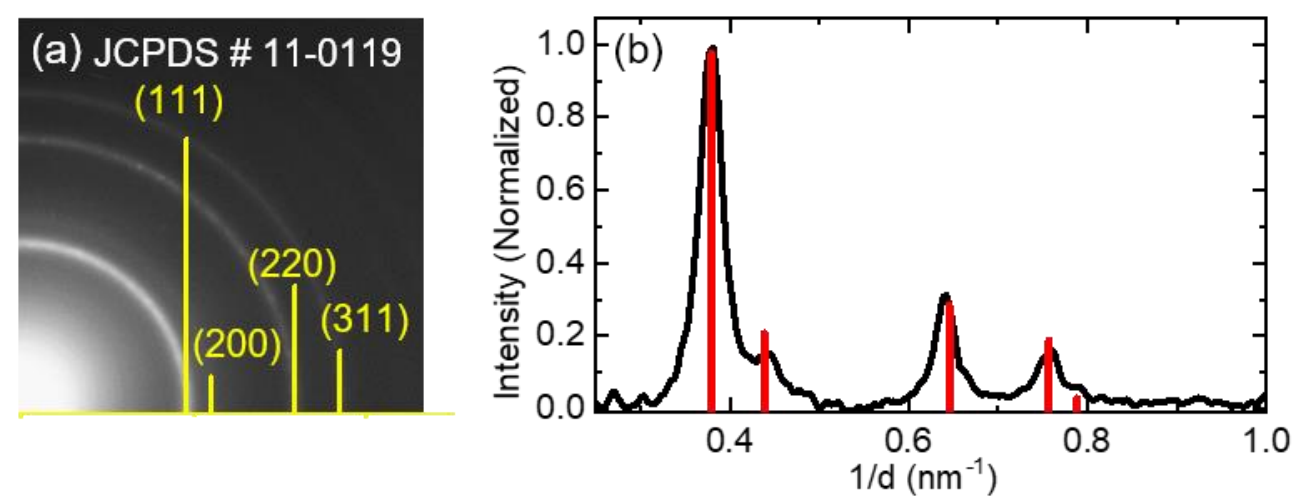

Figure S6. (a) Electron diffraction pattern and (b) its intensity profile of BP nanocrystals grown at $1150^{\circ} \mathrm{C}$.
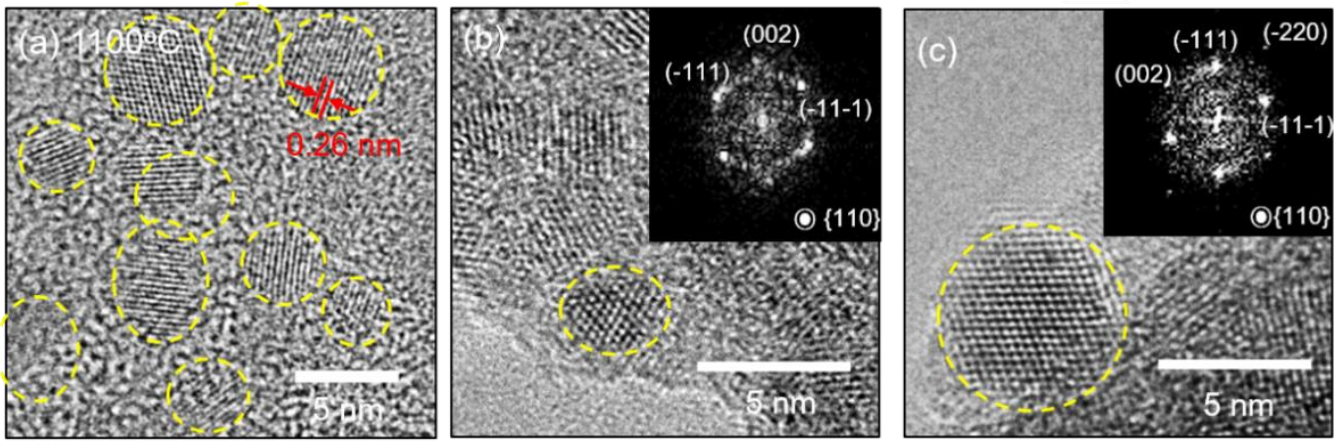

Figure S7. (a-b) High resolution TEM images of BP nanocrystals grown at $1100^{\circ} \mathrm{C}$. Inset of (b) shows FFT image of a BP nanocrystal indicated by a yellow circle. High resolution TEM images of BP nanocrystals grown at (c) $1150^{\circ} \mathrm{C}$.
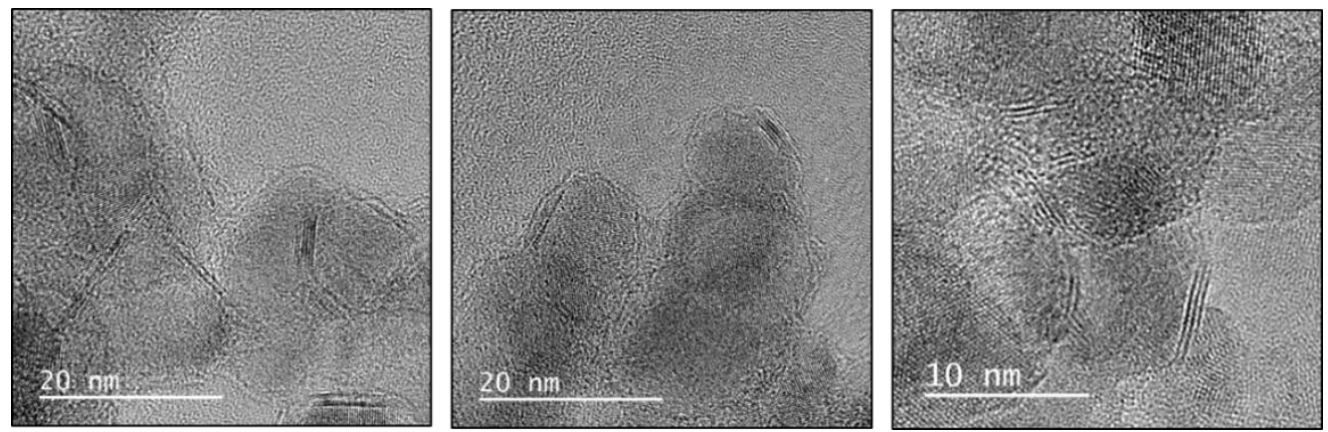

Figure S8. TEM images of BP nanocrystals grown at $1250^{\circ} \mathrm{C}$. 

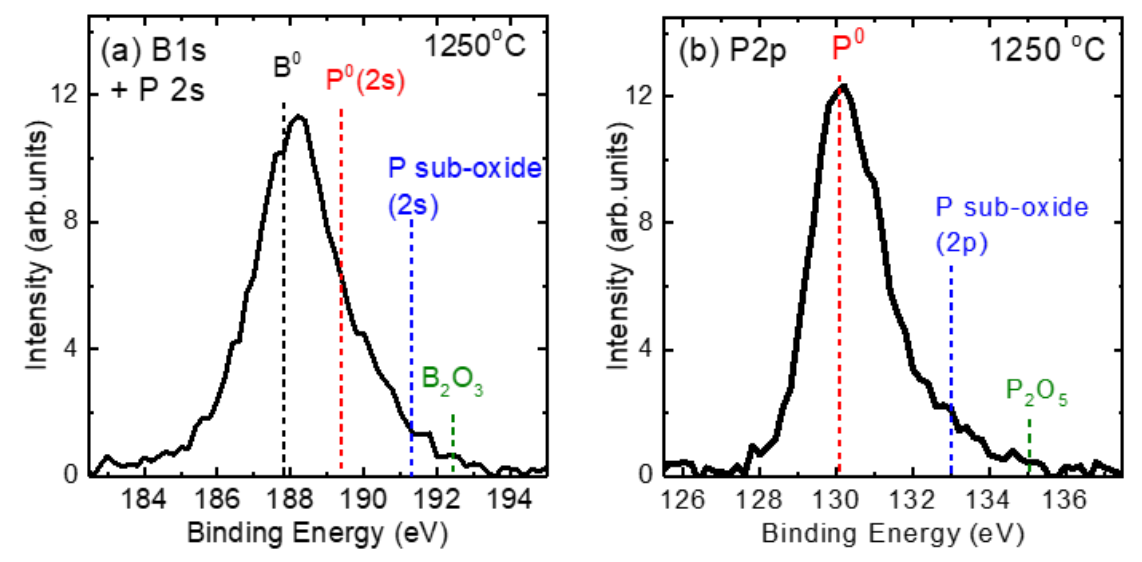

Figure S9. (a) B1s (P 2s) and (b) P 2p XPS spectra of BP nanocrystals grown at $1250^{\circ} \mathrm{C}$.
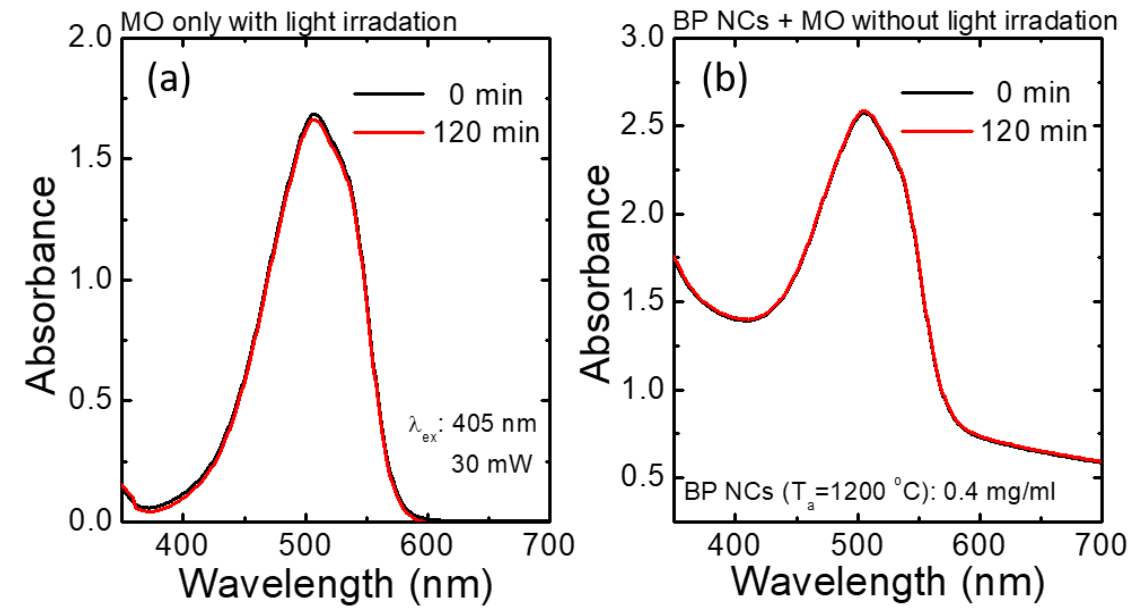

Figure S10. Absorption spectra of a MO solution without BP nanocrystals under light irradiation and a MO and BP nanocrystals mixture solution without light irradiation. 

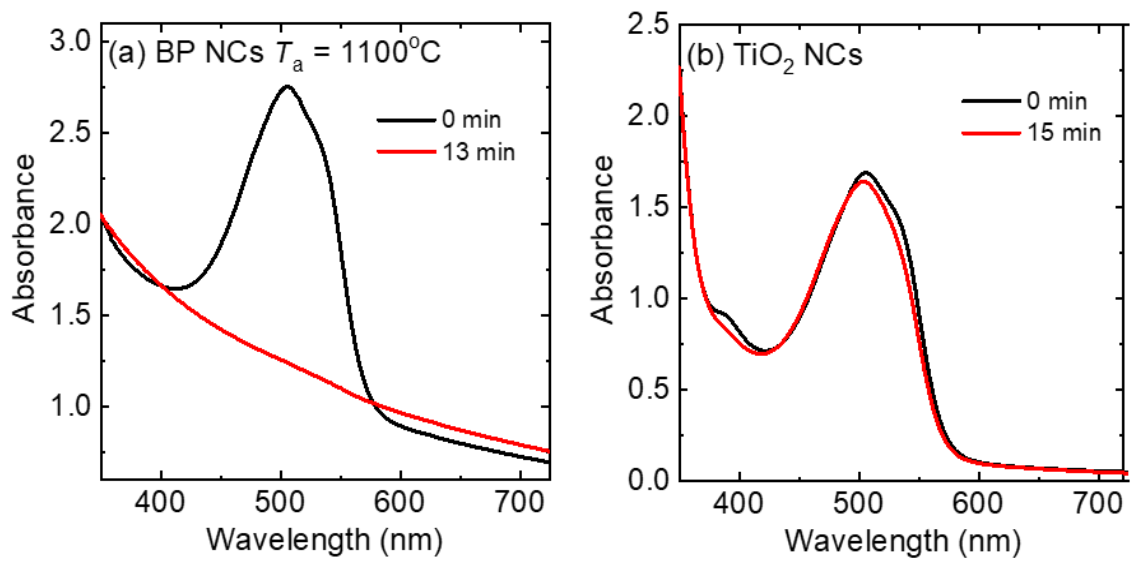

Figure S11. Absorption spectra of (a) a MO and BP nanocrystals mixture solution and (b) a $\mathrm{MO}$ and $\mathrm{TiO}_{2}$ nanocrystals (AEROXIDE P25) mixture solution under $405 \mathrm{~nm}$ light irradiation.
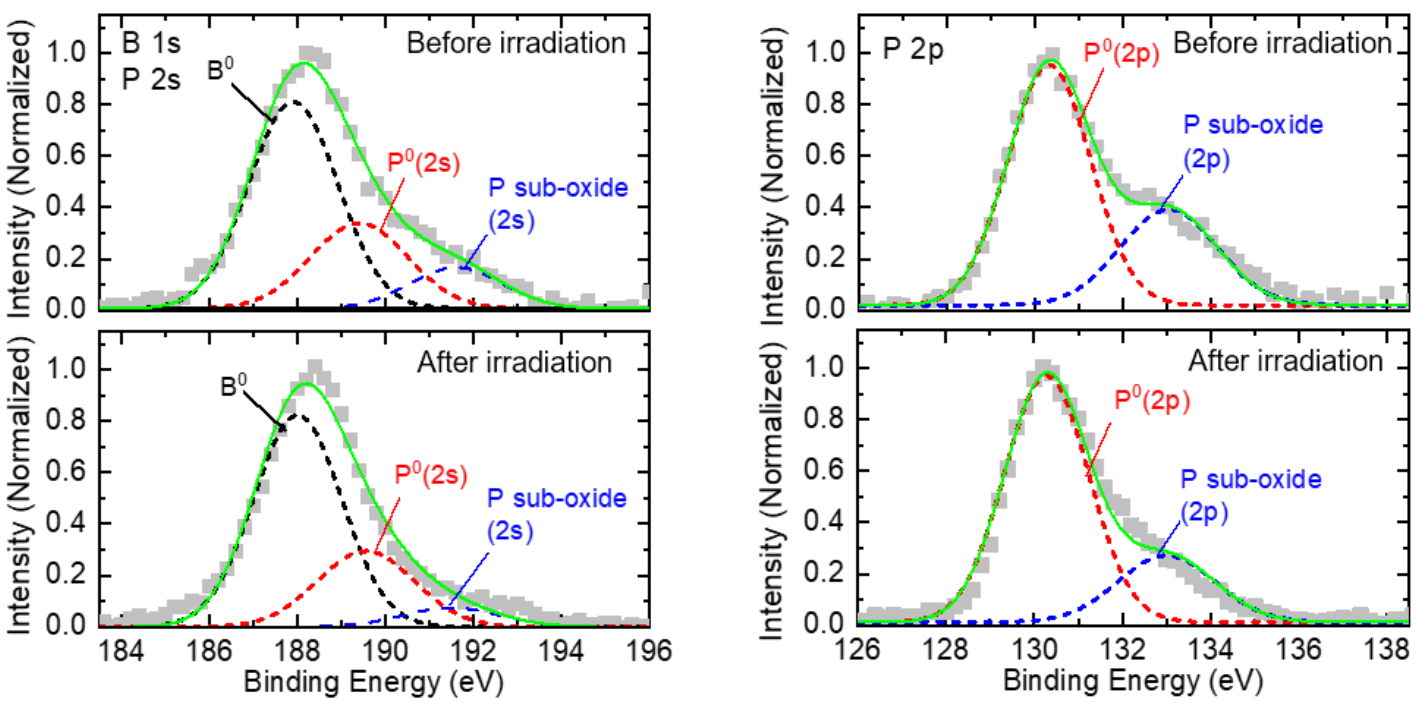

Figure S12. (Left panels) B1s (P 2s) and (Right panels) P 2p XPS spectra of BP nanocrystals grown at $1100^{\circ} \mathrm{C}$ before and after laser irradiation for $15 \mathrm{~min}$. Relative contribution of $\mathrm{P}$ suboxides in the spectra decreases after irradiation. 


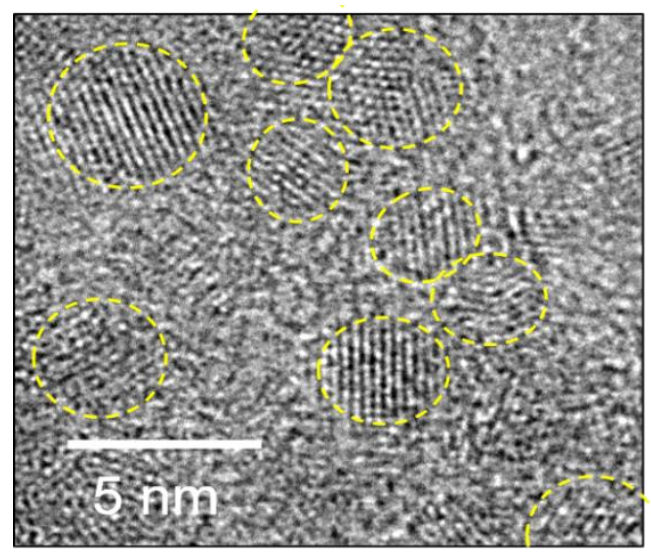

Figure S13. High resolution TEM image of BP nanocrystals after photocatalytic reaction experiments.

References

(1) Perdew, J. P.; Burke, K.; Ernzerhof, M. Generalized Gradient Approximation Made Simple. Phys. Rev. Lett. 1996, 77 (18), 3865-3868.

(2) Perdew, J. P.; Ernzerhof, M.; Burke, K. Rationale for Mixing Exact Exchange with Density Functional Approximations. J. Chem. Phys. 1996, 105 (22), 9982-9985.

(3) Derian, R.; Tokár, K.; Somogyi, B.; Gali, Á.; Štich, I. Optical Gaps in Pristine and Heavily Doped Silicon Nanocrystals: DFT versus Quantum Monte Carlo Benchmarks. J. Chem. Theory Comput. 2017, 13 (12), 6061-6067.

(4) Marsman, M.; Paier, J.; Stroppa, A.; Kresse, G. Hybrid Functionals Applied to Extended Systems. J. Phys. Condens. Matter 2008, 20 (6), 064201.

(5) Sun, J.; Ruzsinszky, A.; Perdew, J. Strongly Constrained and Appropriately Normed Semilocal Density Functional. Phys. Rev. Lett. 2015, 115 (3), 036402.

(6) Krukau, A. V.; Vydrov, O. A.; Izmaylov, A. F.; Scuseria, G. E. Influence of the Exchange Screening Parameter on the Performance of Screened Hybrid Functionals. J. Chem. Phys. 2006, 125 (22), 224106. 
(7) Şahin, H.; Cahangirov, S.; Topsakal, M.; Bekaroglu, E.; Akturk, E.; Senger, R. T.; Ciraci, S. Monolayer Honeycomb Structures of Group-IV Elements and III-V Binary Compounds: First-Principles Calculations. Phys. Rev. B 2009, 80 (15), 155453.

(8) Wu, Y.; Chan, M. K. Y.; Ceder, G. Prediction of Semiconductor Band Edge Positions in Aqueous Environments from First Principles. Phys. Rev. B 2011, 83 (23), 235301.

(9) Plimpton, S. Fast Parallel Algorithms for Short-Range Molecular Dynamics. J. Comput. Phys. 1995, 117 (1), 1-19.

(10) Jorgensen, W. L.; Chandrasekhar, J.; Madura, J. D.; Impey, R. W.; Klein, M. L. Comparison of Simple Potential Functions for Simulating Liquid Water. J. Chem. Phys. 1983, 79 (2), 926-935. 